\title{
ANALYSIS OF SURFACE AND BULK BEHAVIOR IN Ni-Pd ALLOYS
}

\author{
Guillermo Bozzolo ${ }^{a, b, *}$ and Ronald D. Noebe ${ }^{b}$ \\ ${ }^{a}$ Ohio Aerospace Institute, 22800 Cedar Point Rd., Cleveland, OH 44142, USA \\ ${ }^{b}$ NASA Glenn Research Center, Cleveland, OH 44135, USA
}

\begin{abstract}
The most salient features of the surface structure and bulk behavior of Ni-Pd alloys have been studied using the BFS method for alloys. Large-scale atomistic simulations were performed to investigate surface segregation profiles as a function of temperature, crystal face, and composition. Pd enrichment of the first layer was observed in (111) and (100) surfaces, and enrichment of the top two layers occurred for (110) surfaces. In all cases, the segregation profile shows alternate planes enriched and depleted in Pd. In addition, the phase structure of bulk Ni-Pd alloys as a function of temperature and composition was studied. A weak ordering tendency was observed at low temperatures, which helps explain the compositional oscillations in the segregation profiles. Finally, based on atom-by-atom static energy calculations, a comprehensive explanation for the observed surface and bulk features will be presented in terms of competing chemical and strain energy effects.
\end{abstract}

Keywords: surface segregation, modeling, nickel alloys, platinum group, ordering

\section{INTRODUCTION}

A complete understanding of the surfaces of bimetallic systems offers significant challenges because the structure, composition, and thus properties are usually quite different than that expected from the bulk [1]. Because surfaces have an extra degree of freedom compared to the bulk, a number of phenomena unique to surfaces may be observed, such as surface segregation, surface ordering, and reconstruction. Better knowledge, understanding, and eventual exploitation of these phenomena can lead to the development of mixed-metal compounds with potential wide-

his report is a preprint of an article submitted to journal for publication. Because of changes that ray be made before formal publication, this reprint is made available with the understanding lat it will not be cited or reproduced without the ermission of the author. 
spread application in the areas of electrochemistry, microelectronics, and especially catalysis, where mixed metal systems have been found to be much more effective than pure metal surfaces $[1,2]$. The Ni-Pd system is one such example, where surfaces of the binary alloy are more efficient than the surfaces of either of the pure metals at hydrogenation reactions [3-5] and therefore have been studied extensively both experimentally [3-9] and theoretically [10-12]. In spite of the amount of work performed on the Ni-Pd system, there are still questions concerning the surface and near-surface structure in this bimetallic system and the existence of ordered phases.

$\mathrm{Ni}(\mathrm{Pd})$ alloys are an example of a dilute alloy system for which very high segregation of the noble metal to the surface occurs. In fact, it takes very little Pd in solution to significantly enrich the surface, with polycrystalline Ni alloys containing 1 and 5 at.\% Pd consisting of surfaces enriched with 20 and $50 \% \mathrm{Pd}$, respectively [6]. In the study by Miegge et al. [6], the overall enrichment did not seem to extend beyond the second atomic layer and approached the bulk composition by the third layer. Additional analysis of this same Ni-5Pd alloy confirmed that only the surface layer itself was enriched [3]. In fact, significant Pd-enrichment of essentially just the surface layer is consistent with all polycrystalline alloy studies [6,7,9]. The driving force for this behavior can be rationalized by the fact that Pd has a lower surface tension than $\mathrm{Ni}$, which in addition to its larger atomic radius, results in expulsion of the noble metal from the bulk and its segregation to the surface [13].

Studies of single crystal surfaces are consistent with the polycrystalline data in that they also show significant surface enrichment in Pd $[4,8]$. These single crystal studies also revealed additional structural detail, including oscillatory composition profiles in the near-surface region. The first to observe this phenomenon was Derry et al. [8] in a single crystal (100) Ni-50Pd sample. As expected, the surface layer was enriched in $\mathrm{Pd}(80 \%)$. But the first layer below the surface was observed to be $100 \% \mathrm{Ni}$, and the second layer below was enriched once again in Pd (64\%), though less so than the surface layer.

The observed oscillatory behavior is not easily explained in terms of simple segregation models, especially since Ni-Pd is considered a prototypical solid solution alloy over the entire binary alloy composition range with no reported ordered intermetallic phases [14]. Consequently, observation of an oscillatory segregation profile in the dynamical LEED study of (100) Ni-50Pd by Derry et al. [8] is quite unusual [15] and until now has not been fully explained. Furthermore, recent analysis has shown that in addition to the results of Derry et al. [8] where the (100) surface 
layer is rich in Pd and the second layer is essentially pure Ni, results by Michel et al. [4] on (110) surfaces strongly indicate that the first two layers are highly enriched in Pd. No single explanation has yet been presented to explain whether this difference in behavior as a function of surface orientation is consistent and to what extent the known surface behavior is compatible with the behavior of bulk Ni-Pd alloys.

In light of these questions, we have used the Bozzolo-Ferrante-Smith (BFS) method for alloys [16] to study Ni-Pd alloy surfaces, including layer-by-layer compositional analysis of segregation behavior for different crystal faces. Similar analyses were performed for determination of the phase structure of bulk Ni-Pd alloys. From this analytical study we can provide a consistent explanation for the previous experimental findings $[4,8]$ in terms of surface energy arguments, strain effects, and equally important in this case, bulk alloy behavior.

\section{THE BFS METHOD}

The basic features (both surface and bulk) of the Ni-Pd system can be described within the framework of a simple modeling approach grounded in the BFS method for alloys. The BFS method [16] is based on the concept that the energy of formation of a given atomic configuration is the superposition of the individual atomic contributions, $\Delta H=\Sigma \varepsilon_{i}^{T}$. Each contribution $\varepsilon_{i}^{T}$ is the

sum of two terms: a strain energy, $\varepsilon_{i}$, computed in the actual lattice as if every neighbor of the atom $i$ was of the same atomic species $i$, and a chemical energy, $\varepsilon_{i}{ }^{C}$, computed as if every neighbor of the atom $i$ was in an equilibrium lattice site of a crystal of species $i$, but retaining its actual chemical identity. The computation of $\varepsilon_{i}^{S}$, using equivalent crystal theory (ECT) [17], involves three pure elemental properties for atoms of species $i$ : cohesive energy, lattice parameter, and bulk modulus. The chemical energy, $\varepsilon_{i}{ }^{C}$, includes two BFS perturbative parameters, $\Delta_{N i P d}=-0.0396$ $\AA^{-1}$ and $\Delta_{P d N i}=-0.0478 \AA^{-1}$, computed by fitting the BFS calculations to the experimental values of the heat of solution in the dilute limit [18]. A reference chemical energy, $\varepsilon_{i}{ }^{C o}$, is also included to insure a complete decoupling of structural and chemical features. Finally, the strain and chemical energies are linked with a coupling function $g$, which ensures the correct volume dependence of the BFS chemical energy contribution. Therefore, the contribution of atom $i$ to the energy of formation of the system is given by 


$$
\varepsilon_{i}^{T}=\varepsilon_{i}^{S}+g_{i}\left(\varepsilon_{i}^{C}-\varepsilon_{i}^{C_{o}}\right)
$$

Large scale simulations were performed using the Monte Carlo - Nearest Neighbor (MC-NN) approach described in Ref. 19, which is a modified version of the traditional Monte Carlo Metropolis algorithm. An initial, random, structure is generated by assigning $\mathrm{Ni}$ and Pd atoms to rigid (i.e., no individual relaxations) lattice sites in the computational cell with periodic boundary conditions in two directions for surfaces (three directions for bulk cells). Segregation to surfaces or bulk ordering are then simulated by the exchange of randomly chosen pairs of atoms of different atomic species throughout the cell which only occupy nearest-neighbor (NN) sites. The difference in energy of formation of the cell before and after the exchange, $\Delta E$, is used to determine the likelihood that this exchange can take place. BFS was used to calculate the energy at each step. If $\Delta E<0$, the exchange is readily accepted. If not, it is assigned a probability $\exp \left(\Delta E / E_{T h}\right)$, where the available thermal energy, $E_{T h}$, is given by

$E_{T h}=9 N k T\left(\frac{T}{\Theta_{D}}\right)^{3} \int_{0}^{\frac{\Theta_{D}}{T}} \frac{x^{3}}{e^{x}-1} d x$

where $\Theta_{D}$ is the Debye temperature [20] and $k$ is Boltzman's constant. As such, the MC-NN approach does not rely on a rigorous statistical mechanics foundation as traditional Monte CarloMetropolis does. It is, at best, a simple but approximate way to model the evolution of the system [19].

Table 1 lists the necessary parameters for applying the BFS method to the Ni-Pd system. We refer the reader to Ref. 16 for a detailed discussion of the BFS method, its definitions, operational equations and their implementation. All calculations in this work were performed using the adwTools software package [21].

While the methodology allows for a detailed analysis of individual and collective atomic relaxations, no such effects have been included in this initial study. The purpose of the paper is to investigate the balance between strain and chemical effects as driving forces for segregation and ordering, thus focusing on a simple but unified description of bulk and surface behavior. 


\section{RESULTS AND DISCUSSION}

\subsection{Surface and near-surface behavior}

The computed segregation profiles, determined from MC-NN simulations for $\mathrm{Ni}-5 \mathrm{Pd}$ and $\mathrm{Ni}-$ 50Pd alloys, as a function of low-index crystal face, are shown in Fig.1. Each figure includes results for $\mathrm{T}=300$ and $1000 \mathrm{~K}$. Two features are common to all the cases shown: first, the segregation of Pd to the surface layer (top two layers in the case of a (110) face), and second, damped oscillations in the concentration profile for the following subsurface layers. These oscillations, which are usually associated with ordering tendencies in the bulk alloy [15], are more noticeable for the $\mathrm{T}=300 \mathrm{~K}$ profiles. These two basic features are independent of concentration, as all the profiles share a similar Pd-surface enrichment and oscillatory segregation profile near the surface. The segregation profiles also exhibit rapidly decreasing compositional oscillations in the subsequent subsurface layers, approaching the bulk composition after several layers, depending on the bulk concentration of Pd.

While these simulations correctly determine not only the gross behavior but also fine details of segregation in the Ni-Pd system as described in Refs. 4 and 8. Further details on the corresponding driving mechanisms for these observations can also be obtained within the framework of a simple modeling approach. An atom-by-atom energy analysis of a single $\mathrm{Pd}$ atom on or near a $\mathrm{Ni}$ surface can be enough to explain the observed differences between the segregation trends in $\mathrm{Ni}(110)$ and other surfaces. Having shown in Fig. 1 that the (100) and (111) surfaces display the same segregation trends, but different from that of the (110) surface, we limit the comparison to a (100) and a (110) surface. Fig. 2 displays a side view of a $(h k l)$ slab, with the Pd atom located in different planes. We will denote with $\mathrm{Pd}(\mathrm{L} / h k l)$ the configuration where the $\mathrm{Pd}$ atom is located in layer $\mathrm{L}(\mathrm{L}=\mathrm{S}, 1 \mathrm{~b}, 2 \mathrm{~b}$, representing the surface, one atomic layer below the surface, and two layers below the surface, respectively) parallel to a ( $h k l$ ) surface. The role of each 'active' atom (i.e., the $\mathrm{Pd}$ atom and all the $\mathrm{Ni}$ atoms noticeably affected by the presence of the $\mathrm{Pd}$ atom) is quantified by its individual contribution $\left(\varepsilon_{i}^{T}\right)$ to the energy of formation $(\Delta H)$ of the cell and is shown in the corresponding table, along with the BFS strain and chemical contributions that make up $\varepsilon_{i}{ }^{T}$ (Eq. 1). The net difference in energy arising from each active atom with respect to an equivalent atom in a pure $\mathrm{Ni}$ surface, $\Delta E$ /atom, and the number of identical atoms $(m)$ with the same energy in the 
cell are also shown. A reduction in energy occurs when $\Delta \mathrm{E} /$ atom is negative, since it represents a decrease in energy with respect to the contribution of that atom to $\Delta H$ in the $\operatorname{Pd}(\mathrm{L} / h k l)$ cell compared to the contribution of a $\mathrm{Ni}$ atom in the same site in a perfect $\mathrm{Ni}(100)$ or $\mathrm{Ni}(110)$ surface slab (i.e., no Pd present). Whether the entire system is more stable or not depends on the total contribution to the energy by all affected atoms, which is determined by adding the values in the $\Delta \mathrm{E} /$ atom column (once these values are multiplied by the multiplicity factor $m$ ).

For example, Fig. 2.a shows a Pd atom in the (100) surface plane $(\mathrm{Pd}(\mathrm{S} / 100))$. The $\mathrm{Pd}$ atom contributes $0.4597 \mathrm{eV}$ to $\Delta H$, which amounts to a decrease of $0.7286 \mathrm{eV}$ compared to an equivalent site in a pure $\mathrm{Ni}(100)$ surface. Each of the four surface Ni nearest-neighbors (NN) of the Pd atom contribute $1.1693 \mathrm{eV}$ to $\Delta H$, resulting in a total decrease of $0.0760 \mathrm{eV}$ with respect to a pure $\mathrm{Ni}(100)$ surface slab. Other surface Ni atoms, next-nearest-neighbors (NNN) of Pd or beyond, introduce negligible changes in energy, $-0.0001 \mathrm{eV} /$ atom or less, compared to sites in a $\mathrm{Ni}(100)$ surface. Finally, each of the four Ni NN in the $1 \mathrm{~b}$ layer contribute $-0.0027 \mathrm{eV} /$ atom to $\Delta H$, introducing a total decrease in energy of $0.0108 \mathrm{eV}$. $\mathrm{Ni}$ atoms in the $2 \mathrm{~b}$ layer are barely affected by the presence of $\mathrm{Pd}(\mathrm{S})$, thus having a negligible contribution to $\Delta H$. The analysis indicates that all the Pd NN Ni atoms provide favorable contributions to $\Delta H$ resulting in a lower formation energy, but the changes are an order of magnitude smaller than that due to the Pd atom itself. The presence of the one Pd surface atom lowers the energy of the cell by $0.7942 \mathrm{eV}$. This decrease in energy is a result both of the lower surface energy of $\mathrm{Pd}$ and the favorable bonding between $\mathrm{Ni}$ and $\mathrm{Pd}$ atoms (as denoted by the negative contribution to $\Delta H$ from the affected $\mathrm{Ni}$ atoms).

Fig. 2.b shows the Pd atom in the first plane below the $(100)$ surface (without relaxation) $(\mathrm{Pd}(1 \mathrm{~b} / 100))$. The coordination of the Pd atom is nearly bulk-like (with the exception of one missing NNN) and therefore the strain due to the larger Pd atom is too large to introduce a net decrease in energy to the system. The favorable bonding, however, does affect the surrounding Ni atoms introducing a slight decrease in their energy, but not large enough to offset the strain effect due to the embedded Pd atom, resulting in a net gain in energy for the cell of $0.1426 \mathrm{eV}$. A similar situation arises for the $\mathrm{Pd}$ atom located in the second plane below the surface $(\operatorname{Pd}(2 b / 100)$, as shown in Fig. 2.c.

A different picture emerges from a similar analysis of a Ni slab with a (110) termination. Fig. 2.d shows the case of a $\mathrm{Pd}$ atom in a (110) surface site, $(\mathrm{Pd}(\mathrm{S} / 110))$. As in $\mathrm{Pd}(\mathrm{S} / 100)$, most of the energy gain arises from the lower surface energy of Pd, thus guaranteeing Pd enrichment of the 
top layer. The lower coordination of a Pd atom in a $1 \mathrm{~b}$ site (Fig. 2.e), however, leads to an interesting result: the Pd atom, surrounded by just $10 \mathrm{NN}$, finds itself in an environment of almost negligible BFS strain. In other words, the strain energy term, $\varepsilon_{i}{ }^{S}$, as calculated with ECT $[16,17]$, is essentially zero. This fact, added to the favorable nature of the Ni-Pd bonds that are created, lowering the contributions to $\Delta H$ of the surrounding $\mathrm{Ni}$ atoms, leads to an overall decrease in energy $(-0.3124 \mathrm{eV})$ for the cell, although not as large as the one computed for the $\mathrm{Pd}(\mathrm{S} / 110)$ case. It is, however, large enough to favor an enrichment of the $1 \mathrm{~b}$ layer, as observed experimentally [4]. It should be noted that while the insertion of $\mathrm{Pd}$ in the $1 \mathrm{~b}(110)$ surface layer results in energy gains due to a reduced strain, this fact alone is not sufficient to explain the energetically favored $\operatorname{Pd}(1 \mathrm{~b} /$ 110) configuration which, again, arises from a combination of low strain and favorable Ni-Pd bonds. For $\operatorname{Pd}(2 b / 110)$, however, as in the case of $\operatorname{Pd}(1 b / 100)$ and $\operatorname{Pd}(2 b / 100)$, the strain is too large to be offset by a small chemical energy gain, thus resulting in segregation to the upper layers and depletion of $\mathrm{Pd}$ in $2 \mathrm{~b}(110)$.

This analysis, however, is not sufficient to explain the oscillatory nature of the segregation profiles immediately below the surface. But the favorable Ni-Pd bonds observed in the previous analysis does suggest a mechanism for the alternating layer enrichment/depletion of $\mathrm{Pd}$ in the near-surface layers. Consider the (100) surface where the top layer is enriched with Pd. Fig. 3 demonstrates two (idealized) possibilities: a single Pd atom migrates to a site in the $1 \mathrm{~b}$ plane (Fig. 3.a) or the $2 \mathrm{~b}$ plane (Fig. 3.b). Following the notation used in Fig. 2, 'active' atoms are identified by their individual contributions to $\Delta H$ and by the net change in energy with respect to a reference state which, in the examples in Fig. 3, corresponds to a pure Ni(100) or (110) slab with a complete Pd surface layer (i.e., no Pd in layers below the surface) and an additional Pd atom somewhere deep in the bulk. Let $A(L)$ denote an atom of species $A(A=N i, P d)$ in layer $L(L=S, 1 b, 2 b)$. In the first case (Fig. 3.a), the $\mathrm{Pd}(1 \mathrm{~b})$ atom is in a high-strain site $(1.0795 \mathrm{eV})$ due to the almost-full coordination of a $1 \mathrm{~b}$ site in a (100) slab, resulting in a large positive contribution to the energy of the system $(0.7697 \mathrm{eV})$. The Ni atoms in the $1 \mathrm{~b}$ layer that have $\mathrm{Pd}(1 \mathrm{~b})$ as a NN display a substantial reduction in their chemical energy due to the large number of neighboring Pd atoms (the entire surface layer and the isolated $\mathrm{Pd}(1 \mathrm{~b})$ atom). Additional reductions in energy occur from the surrounding $\mathrm{Ni}$ atoms in the $2 \mathrm{~b}$ and $3 \mathrm{~b}$ planes. In spite of these contributions, however, there is a net total increase in the energy of the cell with respect to the case of a pure $\mathrm{Ni}(100)$ slab with a $\mathrm{Pd}$ surface layer $(\Delta E=0.5530 \mathrm{eV})$. 
That is not the case for the $\operatorname{Pd}(2 b)$ atom shown in Fig. 3.b, since the number of Ni-Pd bonds is maximized. The $\mathrm{Pd}(\mathrm{S})$ atoms are barely affected by the $\mathrm{Pd}(2 \mathrm{~b})$ atom which in turn benefits greatly from the (maximized) number of Ni-Pd bonds thus created. The contribution of $\operatorname{Pd}(2 \mathrm{~b})$ is 0.2430 $\mathrm{eV}$, much lower than the $\mathrm{Pd}(1 \mathrm{~b})$ case $(0.7697 \mathrm{eV})$ in spite of the fact that both atoms have almost identical coordination. In the $\operatorname{Pd}(2 b)$ case, the chemical energy more than compensates for the near-bulk strain of the $\operatorname{Pd}(2 b)$ atom. There is a net decrease in energy $(-0.1205 \mathrm{eV})$ for this cell with respect to a pure $\mathrm{Ni}(100)$ slab with a Pd surface layer, thus favoring Pd atoms in this layer. It is also clear that, as a result of the competition between strain and chemical effects, an oscillatory profile emerges, as Pd atoms will populate alternating planes, in spite of the high strain, in order to maximize the gain in chemical energy.

Figs. 3.c-d show the corresponding results for the (110) orientation. The most noticeable feature is the negative contribution to $\Delta H$ of the Pd atom in the $1 \mathrm{~b}$ layer, as seen in Fig. 3.c. Added to the energy gains introduced by the affected surface Pd atoms, as well as the surrounding $\mathrm{Ni}$ atoms, a substantial lowering of the energy with respect to the case when the $\operatorname{Pd}(1 b)$ atom is in the bulk is observed $(-0.3087 \mathrm{eV})$. When the Pd atom is in the $2 \mathrm{~b}$ layer (Fig. 3.d), the same behavior for the affected $\mathrm{Ni}$ atoms is observed (i.e., small negative contributions to the energy of formation). In this case, however, there is a substantial increase in the contribution of the $\operatorname{Pd}(2 b)$ atom, thus leading to a higher total energy for the cell than that obtained when this Pd atom is in the bulk $(0.2087$ $\mathrm{eV})$. As a result, $\mathrm{Pd}(1 \mathrm{~b} / 110)$ is favored, explaining the segregation of Pd to the top two layers for this orientation.

\subsection{Bulk alloy behavior}

The results from the previous section suggest that there is at least a weak tendency for ordering in the Ni-Pd system. However, unlike many binary systems that exhibit a variety of ordered intermetallic phases, the Ni-Pd system is considered an ideal solid solution system [14]. However, experimental evidence for $\mathrm{Ni}-\mathrm{Pd}$ does suggest the existence of at least short-range order effects [22-25]. Anomalous physical properties (electrical, magnetic, heat capacity, and other thermodynamic measurements) as a function of composition strongly hint at the existence of short-range ordering, particularly in compositions rich in Pd [23-25]. Moreover, a detailed study by Lin and Spruiell [22] suggests that local ordering occurs in Ni-50Pd and Ni-75Pd alloys after slow cooling 
or aging at low temperatures (less than $\sim 673 \mathrm{~K}$ ). These "ordered" regions consisted of clusters of like atoms in the $\{100\}$ planes with roughly branched-rod shapes. Within the clusters, like atoms were linked primarily by second nearest neighbors.

Since the BFS method is capable of performing bulk and surface analyses using the same parameter set, the modeling effort was extended to investigate the bulk structure of Ni-Pd alloys in order to further elucidate the possible role of short range order in controlling the near-surface structure of these alloys. For this purpose, large scale, temperature-dependent MC-NN simulations were performed for a variety of Ni-Pd compositions in order to shed light on the bulk structure of such alloys.

Fig. 4 contains interesting MC-NN results concerning the ordering behavior of several Ni-Pd alloys as a function of composition and temperature. Starting with a random initial state, representing a high-temperature alloy, the temperature is lowered in $100 \mathrm{~K}$ steps, letting the system stabilize at any given temperature step. The most striking features of these results are the set of low temperatures obtained for the order-disorder transition for the different stoichiometries and the seemingly unstable ordering patterns observed. The low order-disorder transition temperatures are consistent with the general observation by Lin and Spruiell [22] that it is necessary to age alloys at very low temperatures, less than $675 \mathrm{~K}$, before short range ordering was observed in Ni-50Pd and Ni-75Pd alloys.

Further information on the degree and type of ordering can be obtained from the coordination matrices corresponding to the final states of the simulations (Table 2). The matrix element $a_{i j}\left(b_{i j}\right)$ of the coordination matrix $a(b)$, for NN (NNN), respectively, denotes the probability that an atom of species $i$ has an atom $j$ as a NN (NNN). Consider, in particular, the Ni-75Pd alloy shown in Fig. 4.b.The NN coordination matrix $a$ resembles that for an $\mathrm{L}_{2}$ type ordered alloy. That is not the case for the NNN coordination matrix $b$, which shows a slight departure from the ideal values for $\mathrm{L1}_{2}$ ordering. Visual inspection of the results indicates that this might not be a mere result of the particular simulation performed, but a consequence of the ordering of Pd atoms in rows along the [100] direction, with each Pd atom located at NNN distance from each other. In other words, the system does not appear to be evolving towards the tight ordering implied for an $\mathrm{L}_{2}$ structure, but instead tends to form long strings of Pd atoms in specific directions. These simulations appear to be consistent with the X-ray study performed by Lin and Spruiell [22].

Fig. 4.c shows results of a simulation where the computational cell - representing a Ni-90Pd 
alloy - was cooled down to $\mathrm{T}=50 \mathrm{~K}$. Not surprisingly, the high temperature results show minimum order, almost indistinguishable from a random distribution. As the temperature decreases, particularly below $600 \mathrm{~K}, \mathrm{Ni}$ atoms start to group occupying NNN sites. It is only at room temperature, or below, that more noticeable changes occur, as the $\mathrm{Ni}$ atoms coalesce in what amounts to a small cluster of an $\mathrm{Ll}_{2}$-like $\mathrm{NiPd}_{3}$ structure.

The unusually low temperature for this transition can be traced to the nature of the Ni-Pd bonding and, from a modeling standpoint, can be understood by defining a small set of atomic configurations where a $\mathrm{Ni}$ atom occupies different possible sites relative to a fixed $\mathrm{Ni}$ chain embedded in a Pd alloy: (A) far away from the chain, (B-C) two possible NNN sites and (D-E) two possible NN sites, as defined in Fig. 5.a. After computing the energies of formation of these cells, an energy level spectrum (Fig. 5.b) can be built, highlighting the energy differences (relative to the lowest energy state) between the various configurations. The configurations with the lowest energy are those where the $\mathrm{Ni}$ atom joins the $\mathrm{Ni}$ chain in $\mathrm{NNN}$ sites, the seed of $\mathrm{Ll}_{2}$ ordering, whereas the configurations with substantially higher energy are those where the $\mathrm{Ni}$ atom occupies NN sites, the seed for the formation of a Ni precipitate. The important feature is the proximity of the energy level of configuration A with respect to the other low-lying states, indicating that the bonding between $\mathrm{Ni}$ and $\mathrm{Pd}$ atoms is weak enough so as to barely differentiate states where Ni atoms follow a specific ordering pattern (configurations $\mathrm{B}$ and $\mathrm{C}$ ) or are simply in solution (configuration A). Small thermal excitations can therefore break these bonds which are ultimately established at very low temperatures.

Further understanding of this issue can be obtained from the atom-by-atom analysis of the two competing structures, as schematically shown in Fig. 6. In the first case (Fig. 6.a), two Ni atoms are separated by a distance large enough so as to ignore their mutual interaction. The local environment of each Ni atom is defined by the Ni atom itself and its $12 \mathrm{NN}$ and $6 \mathrm{NNN}$. The contribution of the $\mathrm{Ni}$ atom to $\Delta H$ is $-0.0156 \mathrm{eV}$, and the contribution of each of the neighboring $\mathrm{Pd}$ atoms is $-0.0020 \mathrm{eV} /$ atom (with a negligible contribution from NNN Pd atoms). This results in a net contribution of $-0.0803 \mathrm{eV}$ for the two clusters. Both types of atoms have negative chemical energies. In the case of the neighboring Pd atoms, however, the chemical energy contribution per atom is small $(-0.0020 \mathrm{eV})$. In the second case, shown in Fig. 6.b, where the two Ni atoms occupy NNN sites and the local environment now includes these two atoms and all their $\mathrm{Pd} \mathrm{NN}$ and NNN. This situation is slightly less favorable for each of the two $\mathrm{Ni}$ atoms $(-0.0150 \mathrm{eV})$ with respect to the 
previous case in which they were separated by a large distance. The energy of the Pd NN atoms that are not shared by both Ni atoms remains unchanged. However, the four Pd atoms that are shared by both $\mathrm{Ni}$ atoms have now one additional $\mathrm{Ni}$ neighbor than the others, thus lowering the chemical energy from $-0.0020 \mathrm{eV}$ to $-0.0085 \mathrm{eV}$, making the overall configuration more favorable than the separated $\mathrm{Ni}$ atoms in Fig. 6.a. The energy of formation of the cluster with two Ni atoms in NNN sites is $-0.0967 \mathrm{eV}$, slightly lower than that for the two separate clusters $(-0.0803 \mathrm{eV})$.

There is one point in the previous example worth emphasizing. Although the Ni atoms display a large negative chemical energy contribution when surrounded by $\mathrm{Pd}$ atoms, this contribution is lessened as the number of Ni NNN grows. For example, a single Ni atom increases its energy (i.e., reduces its contribution to the energy of formation) by $0.0006 \mathrm{eV}$ for each $\mathrm{Ni}$ NNN compared to an isolated $\mathrm{Ni}$ atom. While the energy contribution of $\mathrm{Pd}$ atoms favor increased coordination, inducing long range ordering, $\mathrm{Ni}$ atoms do not, instead preferring local ordering. If $\mathrm{Ni}$ atoms also benefited from this increased coordination, then the formation of an $\mathrm{Ll}_{2}$ structure would be more easily promoted, as both $\mathrm{Ni}$ and $\mathrm{Pd}$ atoms would tend to stabilize the structure, thus raising the ordering temperature.

Taken as whole, the description of surface and bulk behavior by means of similar modeling tools helps one understand a variety of dissimilar issues in the framework of a simple, atom-byatom approach. The behavior observed in the surface and near-surface region of Ni-Pd alloys can be rationalized in terms of these subtle interactions between $\mathrm{Ni}$ and Pd atoms. While the segregation of Pd to the Ni surface can be basically explained by strain and surface energy concepts, other near-surface behaviors can only be explained by the additional fact that there is a slight attraction between $\mathrm{Ni}$ and $\mathrm{Pd}$ atoms. For example, the $1 \mathrm{~b}$ layer in a (110) surface is also heavily populated with Pd because the open characteristics of this surface orientation allow chemical effects to have a chance to lower the overall energy of the system. Likewise, the alternating Pd rich and deficient layers near the surface arise for the same reason. This type of behavior is usually associated with ordered alloys [15]. But, as shown in above, there is a weak tendency to order in these alloys since $\mathrm{Pd}$ atoms will lower their energy in the bulk by maximizing the number of Ni nearest-neighbors.

\section{SUMMARY AND CONCLUSIONS}


A self-consistent model was presented for the description of surface and bulk properties of Ni-Pd alloys that not only reproduces existing experimental results but provides a coherent explanation for what was once a series of apparently conflicting data. It was shown that the competition between strain effects (leading to segregation of Pd to the surface) and chemical effects (leading to short-range ordering tendencies), must be taken into account to properly describe the top-layer-only Pd enrichment in (100) and (111) Ni-Pd surfaces vs. the two-layer Pd enrichment observed in a (110) termination and the alternating enrichment and depletion of Pd in near-surface layers. Furthermore, the simulations and atomic analysis of bulk alloys show that the low short-range ordering temperature in bulk Ni-Pd alloys translates into a rapid smoothening of the oscillatory segregation profiles observed in the subsurface layers, especially with increasing temperature.

\section{ACKNOWLEDGMENTS}

Fruitful discussions with N. Bozzolo are gratefully acknowledged. We thank J. Khalil for assistance in performing the MC simulations. Support by Phil Abel and N. Noebe is also appreciated. Financial support for this work was provided through Carol Ginty and the HOTPC project at NASA Glenn Research Center.

\section{REFERENCES}

[1] Rodriguez JA, Surf. Sci. Rep. 1996; 24:223

[2] Bertolini JC, Surf. Rev. Lett. 1996;3:1857

[3] Bertolini JC, Miegge P, Hermann P, Rousset JL, Tardy B, Surf. Sci. 1995; 331-333:651

[4] Michel AC, Lianos L, Rousset JL, Delichere P, Prakash NS, Massardier J, JugneY, Bertolini JC, Surf. Sci. 1998; 416:288

[5] Khanra BC, Bertolini JC, Rousset J, J. Molec. Catal. A: Chem. 1998;129:233

[6] Miegge P, Rousset JL, Tardy B, Massardier J, Bertolini JC, J. Catal. 1994;149:404

[7] Mervyn DA, Baird RJ, Wynblatt P, Surf. Sci. 1979;82:79

[8] Derry GN, McVey CB, Rous PJ, Surf. Sci., 1995;326:59

[9] Stoddart CTH, Moss RL, Pope D, Surf. Sci. 1975; 53:241.

[10] Christensen A, Ruban AV, Skriver HL, Surf. Sci. 1997; 383:235 
[11] Filhol JS, Simon D, Sautet P, Surf. Sci. 2001;472:L139

[12] Rousset JL, Bertolini JC, Miegge P, Phys. Rev. B 1996; 53:4947

[13] Wynblatt P, Ku RC, Surf. Sci. 1977;65:511

[14] Binary Alloy Phase Diagrams, Second Edition, ASM International, Metals Park, OH, 1990.

[15] Tersoff J, Phys. Rev. B 1990;42:10965

[16] Bozzolo G, Noebe RD, Ferrante J, Amador C, J. Comput.-Aided Mater. Design 1999;6:1

[17] Smith JR, Perry T, Banerjea A, Ferrante J, Bozzolo G, Phys. Rev. B 1991;44:6444, Bozzolo

G, Ferrante J, Rodriguez AM, J. Computer-Aided Mater. Design 1993;1:285.

[18] Hultgren RD, Desai PD, Hawkins DT, Gleiser M and Kelley KK, Selected Values of the Thermodynamic Properties of Binary Alloys, American Society for Metals, Metals Park, $\mathrm{OH}$, 1973.

[19] Bozzolo G, Khalil J, Noebe RD, Comp. Mat. Sci. 2002;24:457

[20] Burns DG, Solid State Physics, Academic Press, New York, 1985.

[21] adwTools v2.5, Morse J, Bozzolo G, Khalil J, Noebe RD, Abel P, Good B, Ohio Aerospace Institute and NASA GRC, 2002.

[22] Lin W, Spruiell JE, Acta Metall. 1971;19:451

[23] Schwerdtfeger K, Muan A, Acta Metall. 1965;13: 509

[24] Bidwell LR, Speiser R, Acta Metall. 1965, 13, 61

[25] Bingham RE, Brooks C, J. Phys. Chem. Solids 1969;30:2365 


\section{TABLE CAPTIONS}

Table 1: Experimental values for the lattice parameter, cohesive energy, and bulk modulus for the fcc phases of $\mathrm{Ni}$ and $\mathrm{Pd}$, and the resulting Equivalent Crystal Theory (ECT) parameters $\mathrm{p}, \alpha, l$ and $\lambda$ (see text and Ref. 17).

Table 2: Nearest-neighbors and next-nearest-neighbors coordination matrices for Ni-50Pd, Ni-75 Pd and Ni-90Pd alloys shown in Fig. 4 ( $\mathrm{T}=300 \mathrm{~K}$ ).

\section{FIGURE CAPTIONS:}

Fig. 1: Segregation profiles for Ni-50Pd and Ni-5Pd alloys for the lowest index surfaces. Solid and dashed lines correspond to profiles obtained at $1000 \mathrm{~K}$ and $300 \mathrm{~K}$, respectively. The horizontal axis denotes the plane ( $S=$ surface plane, $1 \mathrm{~b}=$ first plane below $S$, etc.) and the vertical axis indicates the concentration of $\mathrm{Ni}$ or $\mathrm{Pd}$ (in at.\%).

Fig. 2: a-c) Side view of a Ni (100) surface and d-f) a Ni (110) surface. Circles denote atoms in the plane of the page, while squares denote atoms one plane in and one plane out of the page. The $\mathrm{Pd}$ atom is indicated with a large black disk. Nearby Ni atoms (at NN or NNN distance from the $\mathrm{Pd}$ atom) are indicated with grey symbols. Other $\mathrm{Ni}$ atoms are indicated with open circles and squares. Each atom is labeled with its contribution to the total energy of formation of the slab, and the difference in energy with respect to a pure $\mathrm{Ni}(100)$ slab (in the corresponding tables). The total energy difference $\Delta E$ of the cell compared to a pure Ni surface is denoted for each cell.

Fig. 3: Side view of a Ni (100) slab with a Pd surface layer and a Pd atom in the a) 1 b layer and b) $2 b$ layer. c) and d) show similar results for a Ni(110) surface. Circles denote atoms in the plane of the page, while squares denote atoms one plane in and one plane out of the page. Pd atoms are indicated with large black disks or squares. Nearby Ni atoms (at NN or NNN distance from Pd atoms) are indicated with grey symbols. Other $\mathrm{Ni}$ atoms are indicated with open circles and squares. Energy contributions of individual atoms to the energy of formation are indicated in the 
corresponding tables.

Fig. 4: Atomic distributions during a MC-NN simulation for Ni-50Pd, Ni-75Pd, and Ni-90Pd alloys. Starting from an initial, high temperature computational cell, the temperature is lowered to a final temperature of $300 \mathrm{~K}$ for Ni-50 Pd and Ni-75 Pd, and $50 \mathrm{~K}$ for Ni-90Pd. Equilibrium states at intermediate temperatures are shown, highlighting the range of temperature where the disorderorder transition takes place. $\mathrm{Ni}$ and $\mathrm{Pd}$ atoms are denoted with dark and open disks, respectively.

Fig. 5: (a) (001) view of bulk configurations showing some possible locations of a Ni atom (grey solid squares) in the vicinity of a Ni-chain (black solid squares) in a Pd (open symbols) alloy: (A) away from the tip of the chain, (B-C) occupying a NNN site and (D-E) occupying a NN site. (b) Energy level spectrum showing the differences in energy of formation (in $\mathrm{eV}$ ) between these configurations and the lowest energy state.

Fig. 6: (a) Two Ni atoms (black squares) in a Pd (open symbols) alloy separated by a large distance and their respective local environment (shaded area, containing the Pd NN to the central Ni atom). (b) Same, but with the two Ni atoms located in NNN sites. 


\begin{tabular}{cccccccc}
\hline $\begin{array}{c}\text { Lattice } \\
\text { parameter } \\
(\AA)\end{array}$ & $\begin{array}{c}\text { Cohesive } \\
\text { energy } \\
(\mathrm{eV})\end{array}$ & $\begin{array}{c}\text { Bulk } \\
\text { modulus } \\
(\mathrm{GPa})\end{array}$ & $\mathrm{p}$ & $\begin{array}{c}\alpha \\
\left(\AA^{-1}\right)\end{array}$ & $\begin{array}{c}l \\
(\AA)\end{array}$ & $\begin{array}{c}\lambda \\
(\AA)\end{array}$ \\
\hline $\mathrm{Ni}$ & 3.524 & 4.435 & 148.81 & 6 & 3.015 & 0.270 & 0.7587 \\
$\mathrm{Pd}$ & 3.89 & 3.94 & 156.23 & 8 & 3.612 & 0.237 & 0.666 \\
\hline
\end{tabular}

Table 1 


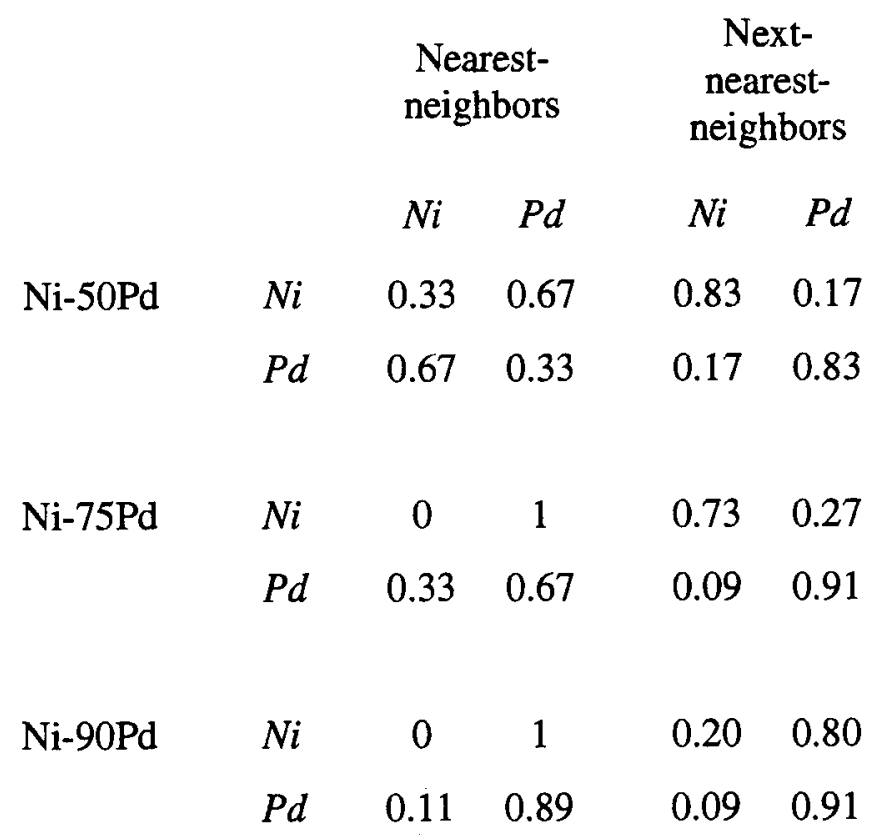

Table 2 

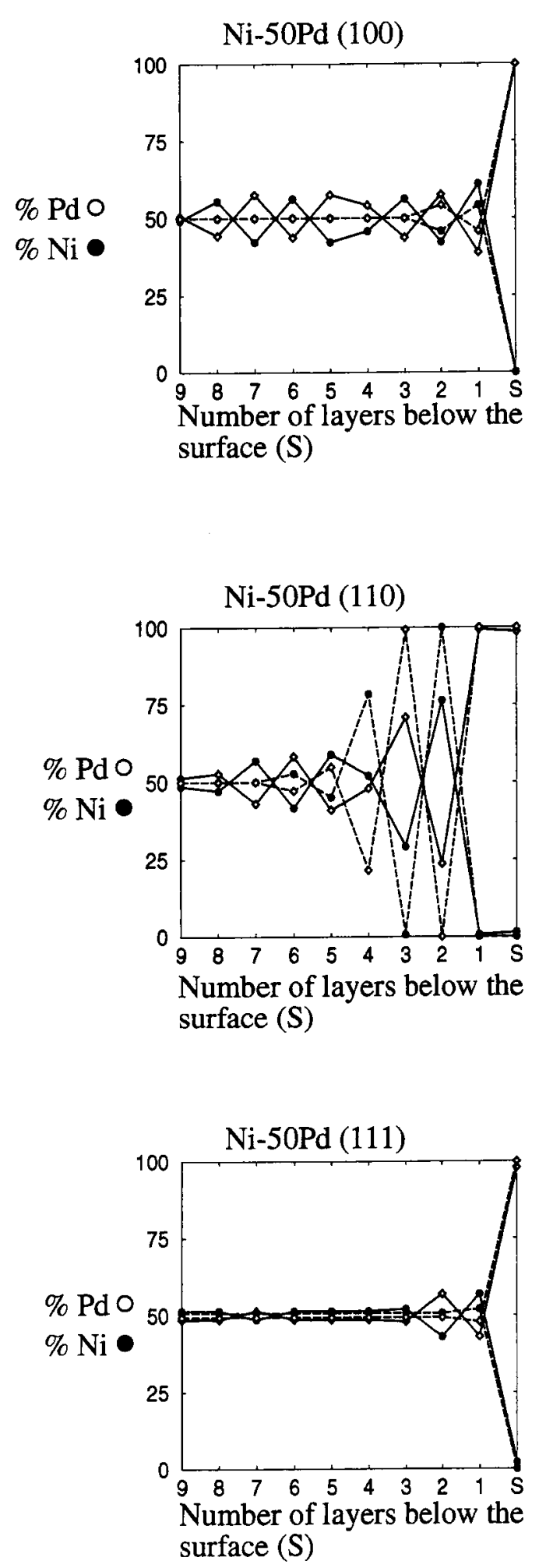
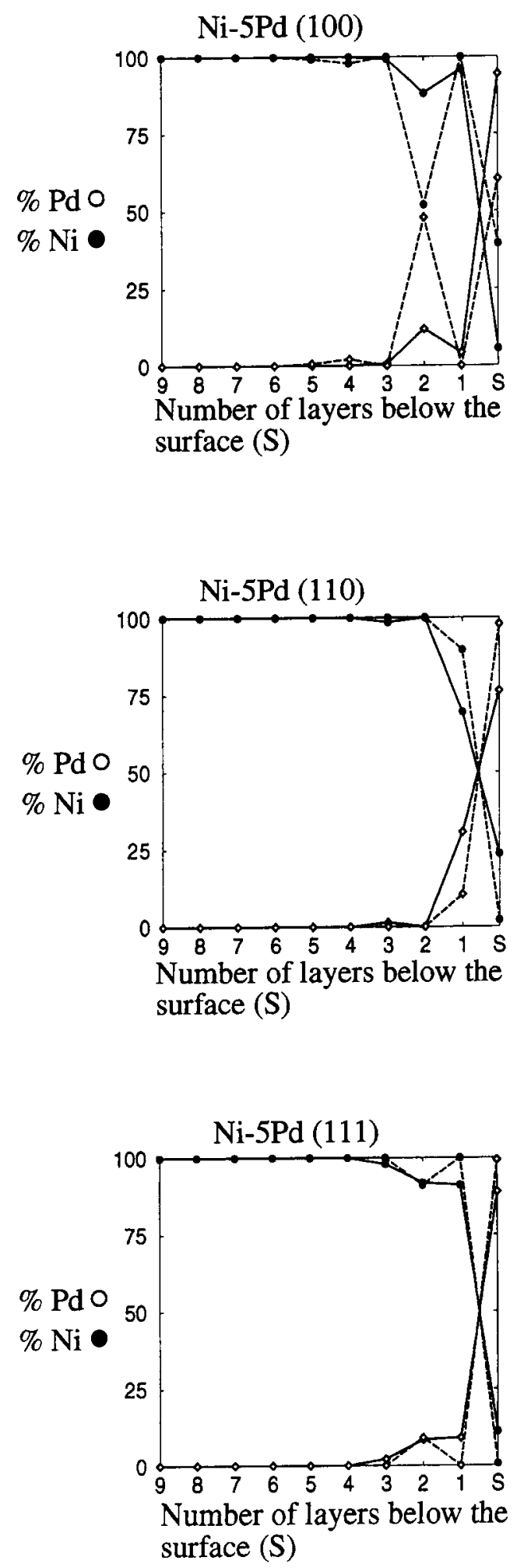

Fig. 1 


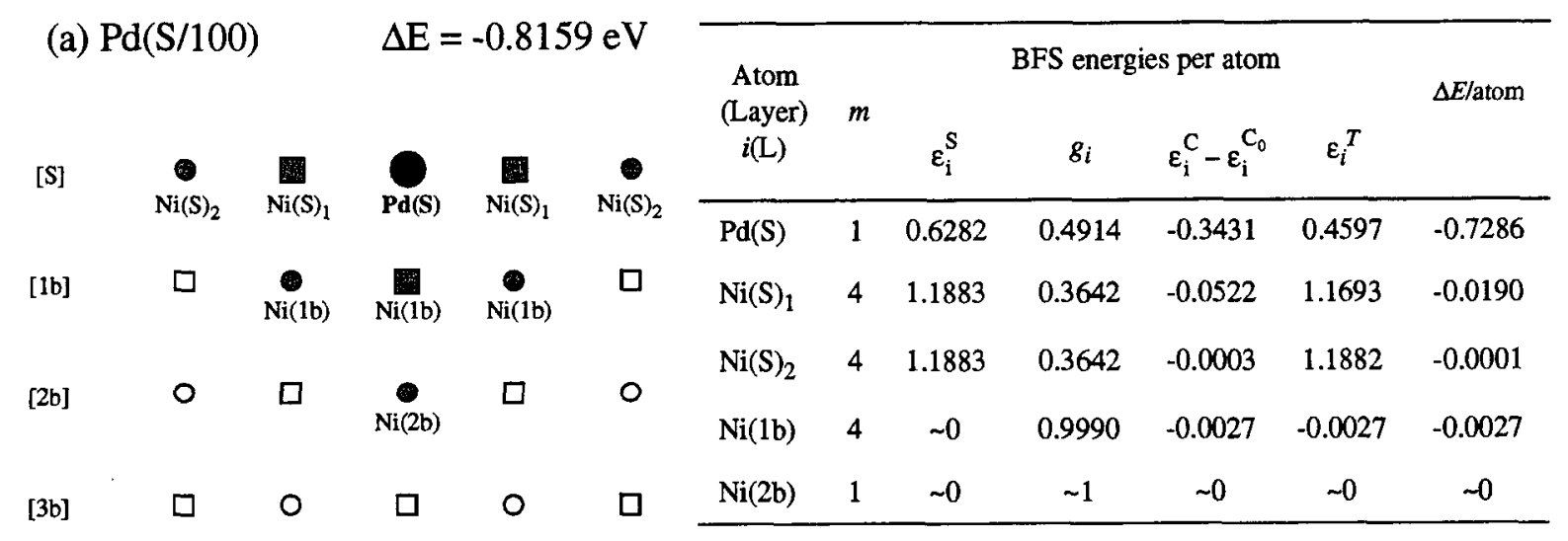

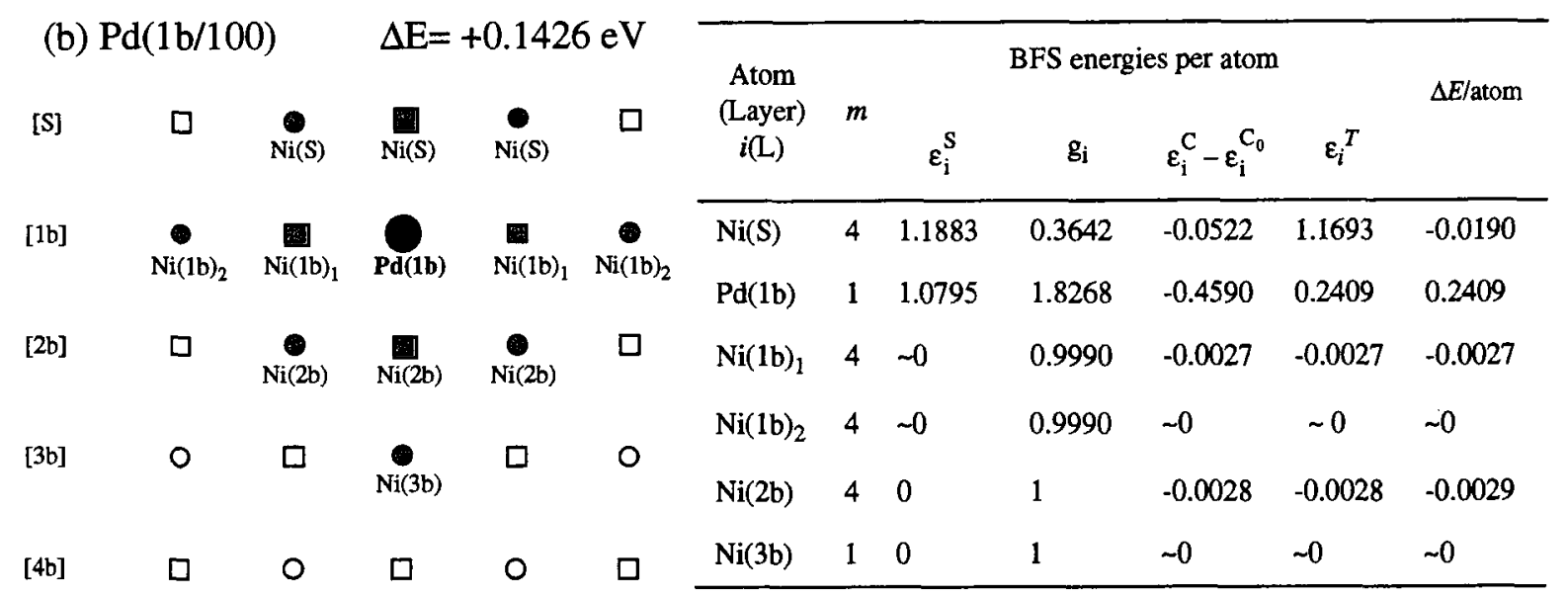

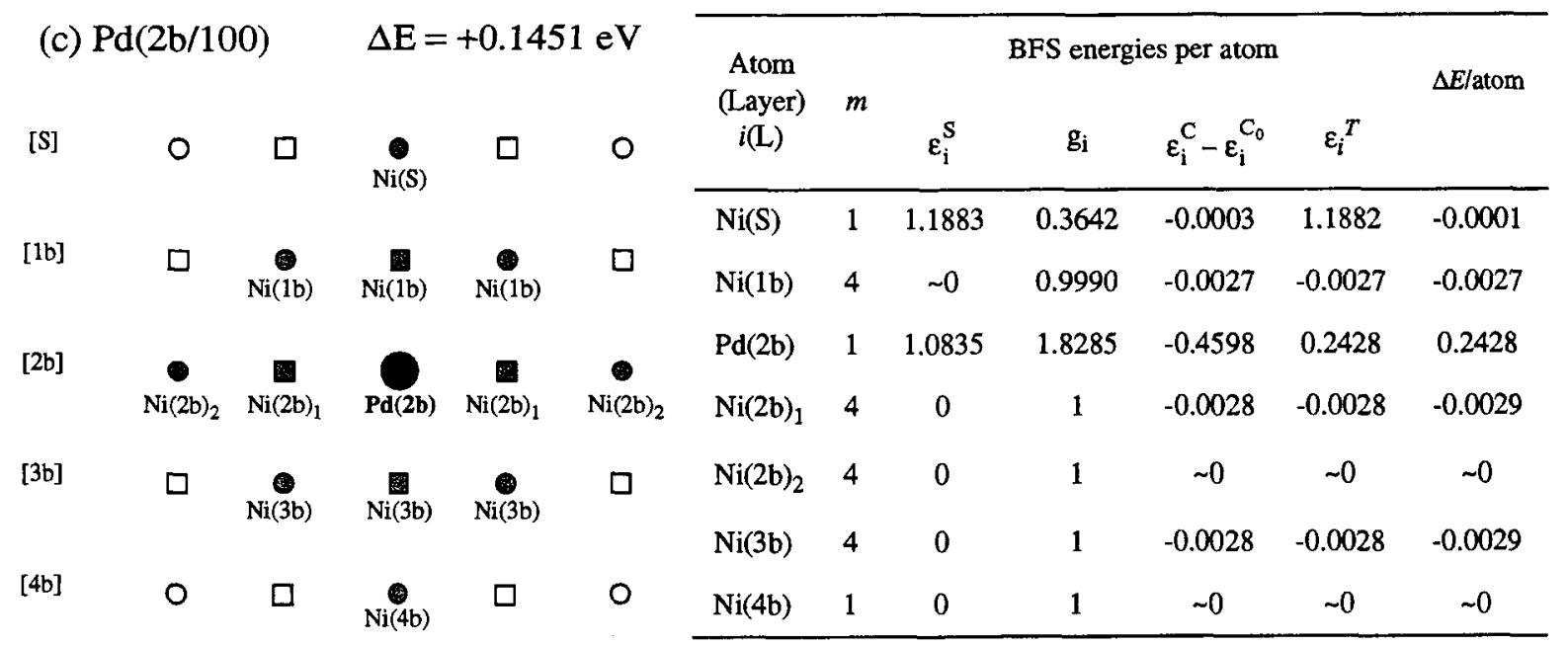

Fig. 2 


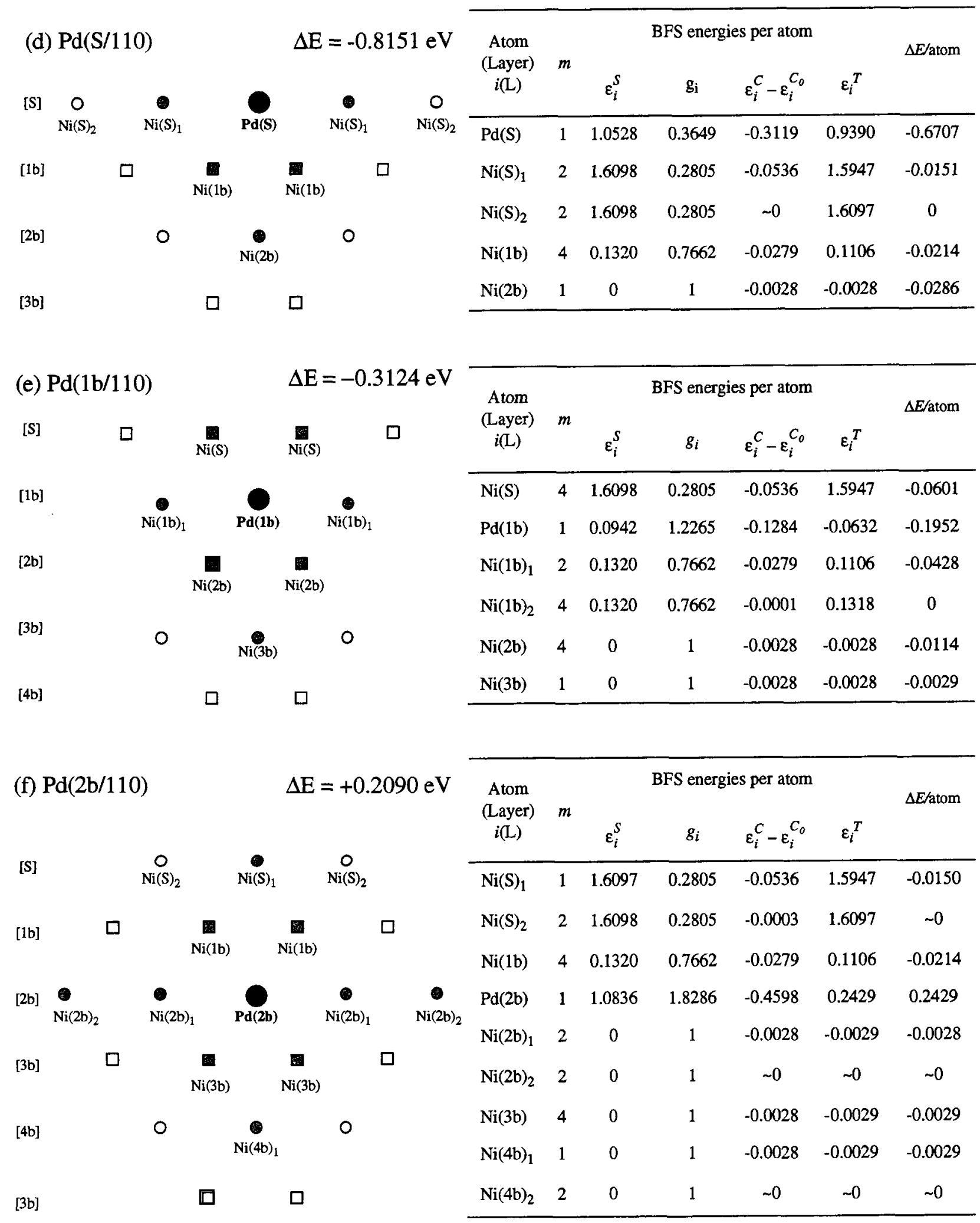

Fig. 2 (continued) 
(a) $\operatorname{Pd}(S / 100)+\operatorname{Pd}(1 b)$

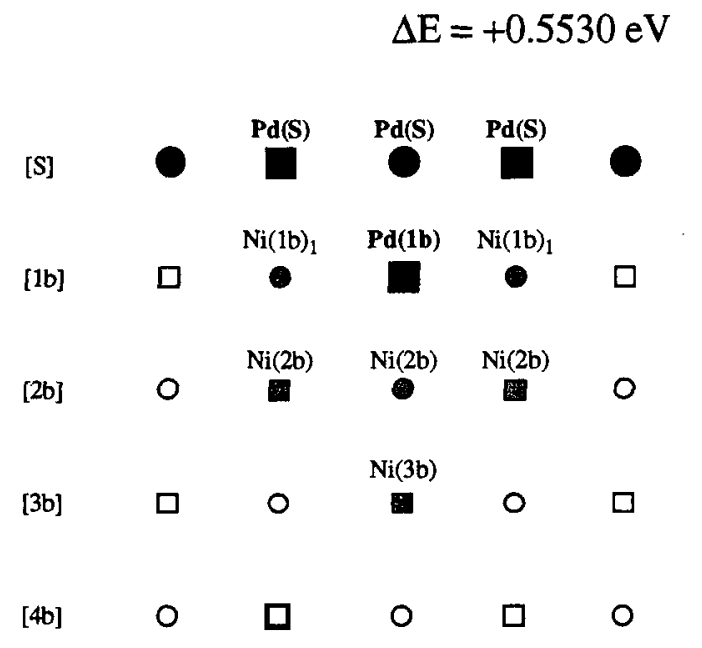

\begin{tabular}{|c|c|c|c|c|c|c|}
\hline \multirow{2}{*}{$\begin{array}{c}\text { Atom } \\
\text { (Layer) } \\
i(\mathrm{~L})\end{array}$} & \multirow{2}{*}{$m$} & \multicolumn{4}{|c|}{ BFS energies per atom } & \multirow{2}{*}{$\Delta E /$ atom } \\
\hline & & $\varepsilon_{i}^{S}$ & $g_{i}$ & $\varepsilon_{i}^{C}-\varepsilon_{i}^{C_{0}}$ & $\varepsilon_{i}^{T}$ & \\
\hline $\operatorname{Pd}(\mathbf{S})$ & 4 & 0.6282 & 0.4914 & -0.1307 & 0.5640 & 0.0212 \\
\hline $\operatorname{Pd}(1 b)$ & 1 & 1.0795 & 1.8268 & -0.1696 & 0.7697 & 0.8210 \\
\hline $\mathrm{Ni}(1 \mathrm{~b})_{1}$ & 4 & $\sim 0$ & 0.9990 & -0.0845 & -0.0844 & -0.0331 \\
\hline $\mathrm{Ni}(1 \mathrm{~b})_{2}$ & 4 & $\sim 0$ & 0.9990 & -0.0515 & -0.0515 & -0.0002 \\
\hline $\mathrm{Ni}(2 \mathrm{~b})$ & 4 & 0 & 1 & -0.0029 & -0.0029 & -0.0029 \\
\hline $\mathrm{Ni}(3 b)$ & 1 & 0 & 1 & -0 & $\sim 0$ & $\sim 0$ \\
\hline Ni(bulk) & 1 & 0 & 1 & 0 & 0 & -0.2428 \\
\hline $\mathrm{Ni}(\mathrm{NN})$ & 12 & 0 & 1 & 0 & 0 & +0.0029 \\
\hline $\mathrm{Ni}(\mathrm{NNN})$ & 6 & 0 & 1 & 0 & 0 & $\sim 0$ \\
\hline
\end{tabular}

(b) $\operatorname{Pd}(S / 100)+\operatorname{Pd}(2 b)$

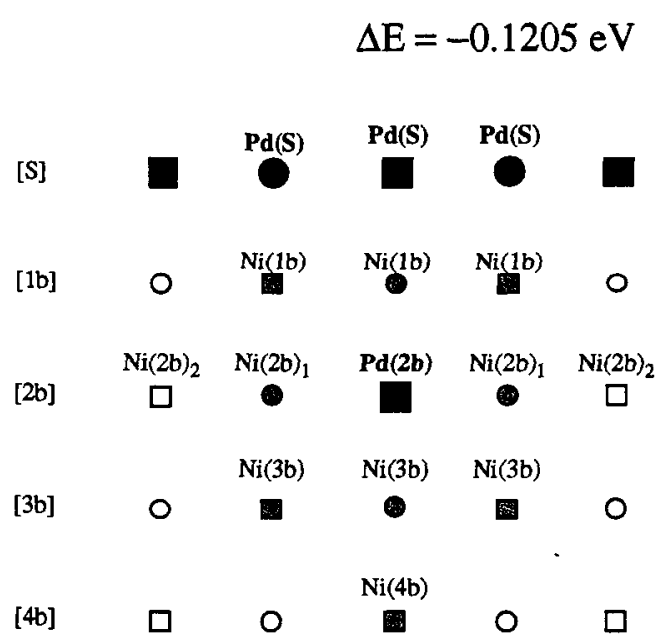

\begin{tabular}{lcccccc}
\hline $\begin{array}{c}\text { Atom } \\
\text { (Layer) } \\
i(\mathrm{~L})\end{array}$ & $m$ & $\varepsilon_{i}^{S}$ & $g_{i}$ & $\varepsilon_{i}^{C}-\varepsilon_{i}^{C_{0}}$ & $\varepsilon_{i}^{T}$ & \\
\hline $\mathrm{Pd}(\mathrm{S})$ & 1 & 0.6282 & 0.4914 & -0.1738 & 0.5429 & 0.0001 \\
$\mathrm{Ni}(\mathrm{lb})$ & 4 & $\sim 0$ & 0.9990 & -0.0845 & -0.0844 & -0.0331 \\
$\mathrm{Pd}(2 \mathrm{~b})$ & 1 & 1.0835 & 1.8285 & -0.4597 & 0.2430 & 0.2430 \\
$\mathrm{Ni}(2 \mathrm{~b})_{1}$ & 4 & 0 & 1 & -0.0029 & -0.0029 & -0.0029 \\
$\mathrm{Ni}(2 \mathrm{~b})_{2}$ & 4 & 0 & 1 & $\sim 0$ & $\sim 0$ & $\sim 0$ \\
$\mathrm{Ni}(3 \mathrm{~b})$ & 4 & 0 & 1 & -0.0029 & -0.0029 & -0.0029 \\
$\mathrm{Ni}(4 \mathrm{~b})$ & 1 & 0 & 1 & $\sim 0$ & $\sim 0$ & $\sim 0$ \\
$\mathrm{Ni}(\mathrm{bulk})$ & 1 & 0 & 1 & 0 & 0 & -0.2428 \\
$\mathrm{Ni}(\mathrm{NN})$ & 12 & 0 & 1 & 0 & 0 & +0.0029 \\
$\mathrm{Ni}(\mathrm{NNN})$ & 6 & 0 & 1 & 0 & 0 & $\sim 0$ \\
\hline
\end{tabular}

Fig. 3 
(c) $\mathrm{Pd}(\mathrm{S} / 110)+\mathrm{Pd}(1 \mathrm{~b})$

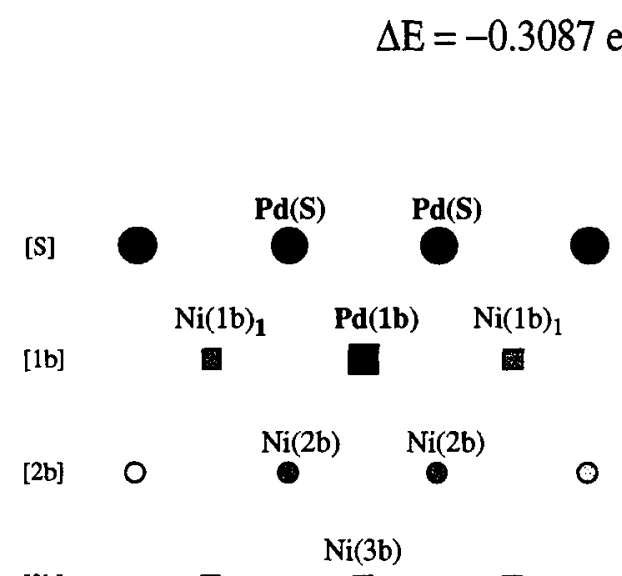

(d) $\operatorname{Pd}(S / 110)+P d(2 b)$

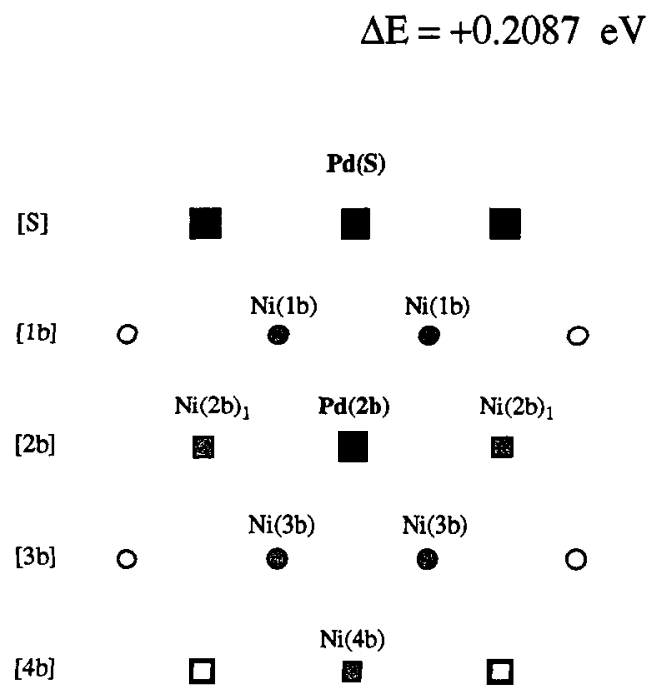

\begin{tabular}{|c|c|c|c|c|c|c|}
\hline \multirow{2}{*}{$\begin{array}{c}\text { Atom } \\
\text { (Layer) } \\
i(\mathrm{~L})\end{array}$} & \multirow{2}{*}{$m$} & \multicolumn{4}{|c|}{ BFS energies per atom } & \multirow{2}{*}{$\Delta E /$ atom } \\
\hline & & $\varepsilon_{i}^{S}$ & $g_{i}$ & $\varepsilon_{i}^{C}-\varepsilon_{i}^{C_{0}}$ & $\varepsilon_{i}^{T}$ & \\
\hline $\operatorname{Pd}(S)$ & 4 & 1.0528 & 0.3649 & -0.1787 & 0.9876 & 0.0162 \\
\hline $\operatorname{Pd}(1 b)$ & 1 & 0.0942 & 1.2265 & -0.1116 & -0.0427 & -0.1020 \\
\hline $\mathrm{Ni}(1 \mathrm{~b})_{1}$ & 2 & 0.1320 & 0.7662 & -0.1105 & 0.0473 & -0.0120 \\
\hline $\mathrm{Ni}(1 \mathrm{~b})_{2}$ & 2 & 0.1320 & 0.7662 & -0.0949 & 0.0592 & -0.0001 \\
\hline $\mathrm{Ni}(2 \mathrm{~b})$ & 4 & 0 & 1 & -0.0120 & -0.0120 & -0.0091 \\
\hline $\mathrm{Ni}(3 \mathrm{~b})_{1}$ & 1 & 0 & 1 & -0.0029 & -0.0029 & -0.0029 \\
\hline $\mathrm{Ni}(3 \mathrm{~b})_{2}$ & 2 & 0 & 1 & $\sim 0$ & $\sim 0$ & $\sim 0$ \\
\hline Ni(bulk) & 1 & 0 & 1 & 0 & 0 & -0.2428 \\
\hline $\mathrm{Ni}(\mathrm{NN})$ & 12 & 0 & 1 & 0 & 0 & +0.0029 \\
\hline $\mathrm{Ni}(\mathrm{NNN})$ & 6 & 0 & 1 & 0 & 0 & $\sim 0$ \\
\hline
\end{tabular}

\begin{tabular}{|c|c|c|c|c|c|c|}
\hline \multirow{2}{*}{$\begin{array}{c}\text { Atom } \\
\text { (Layer) } \\
i(\mathrm{~L})\end{array}$} & \multirow{2}{*}{$m$} & \multicolumn{4}{|c|}{ BFS energies per atom } & \multirow{2}{*}{$\Delta E$ /atom } \\
\hline & & $\varepsilon_{i}^{S}$ & $g_{i}$ & $\varepsilon_{i}^{C}-\varepsilon_{i}^{C_{0}}$ & $\varepsilon_{i}^{T}$ & \\
\hline $\operatorname{Pd}(S)$ & 1 & 1.0528 & 0.3649 & -0.1787 & 0.9876 & 0.0162 \\
\hline $\mathrm{Ni}(1 \mathrm{~b})$ & 4 & 0.1320 & 0.7662 & -0.1105 & 0.0473 & -0.0120 \\
\hline $\operatorname{Pd}(2 b)$ & 1 & 1.0837 & 1.8285 & -0.3675 & 0.4118 & 0.4147 \\
\hline $\mathrm{Ni}(2 \mathrm{~b})_{1}$ & 2 & 0 & 1 & -0.0120 & -0.0120 & -0.0091 \\
\hline $\mathrm{Ni}(2 \mathrm{~b})_{2}$ & 2 & 0 & 1 & -0.0029 & -0.0029 & 0 \\
\hline $\mathrm{Ni}(3 \mathrm{~b})$ & 4 & 0 & 1 & -0.0029 & -0.0029 & -0.0029 \\
\hline $\mathrm{Ni}(4 \mathrm{~b})$ & 1 & 0 & 1 & $\sim 0$ & $\sim 0$ & 0 \\
\hline Ni(bulk) & 1 & 0 & 1 & 0 & 0 & -0.2428 \\
\hline $\mathrm{Ni}(\mathrm{NN})$ & 12 & 0 & 1 & 0 & 0 & +0.0029 \\
\hline $\mathrm{Ni}(\mathrm{NNN})$ & 6 & 0 & 1 & 0 & 0 & $\sim 0$ \\
\hline
\end{tabular}

Fig. 3 (continued) 
(a)

Ni-50Pd

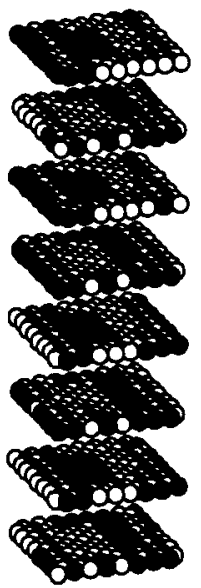

$\mathrm{T}=800 \mathrm{~K}$

(b)

Ni-75Pd

(c)
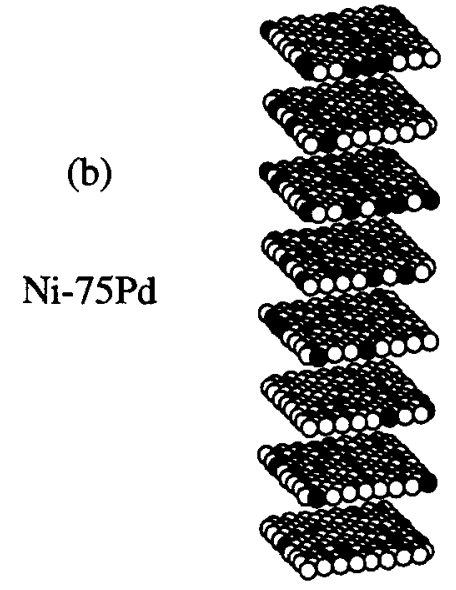

$\mathrm{T}=800 \mathrm{~K}$

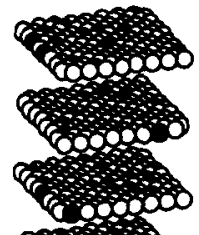

Ni-90Pd

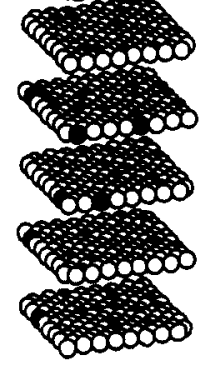

$\mathrm{T}=800 \mathrm{~K}$

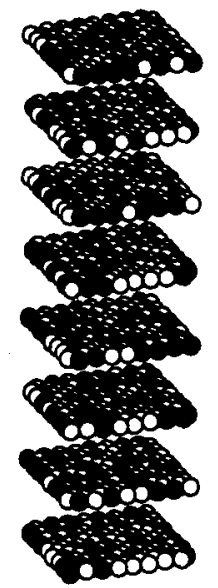

$\mathrm{T}=600 \mathrm{~K}$

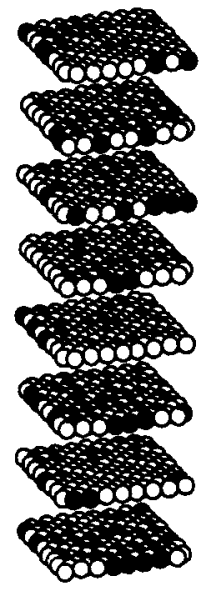

$\mathrm{T}=700 \mathrm{~K}$
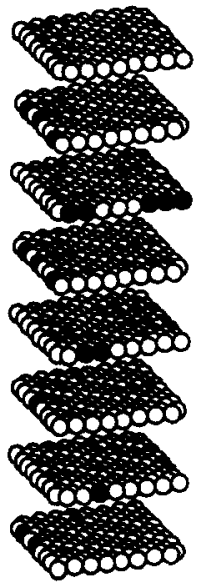

$\mathrm{T}=600 \mathrm{~K}$

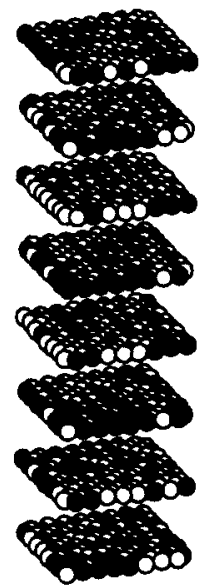

$\mathrm{T}=500 \mathrm{~K}$

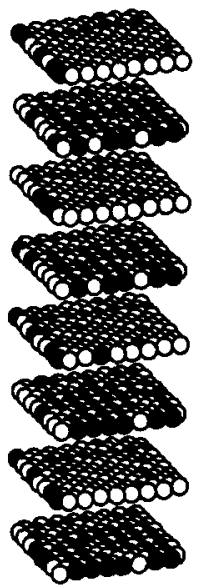

$\mathrm{T}=600 \mathrm{~K}$
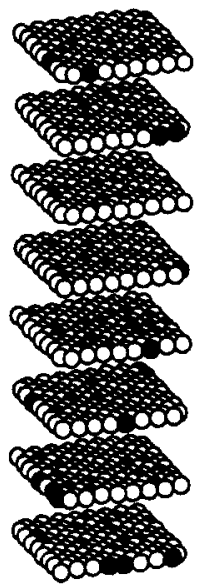

$\mathrm{T}=300 \mathrm{~K}$

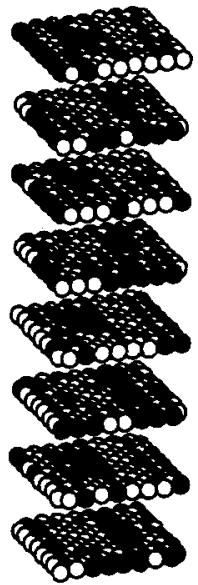

$\mathrm{T}=300 \mathrm{~K}$

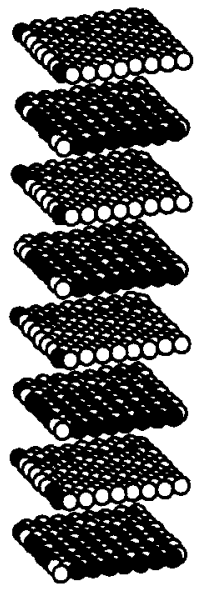

$\mathrm{T}=300 \mathrm{~K}$

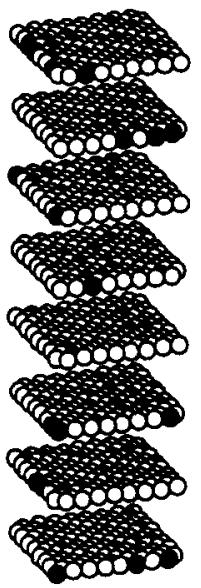

$\mathrm{T}=50 \mathrm{~K}$

Fig. 4 

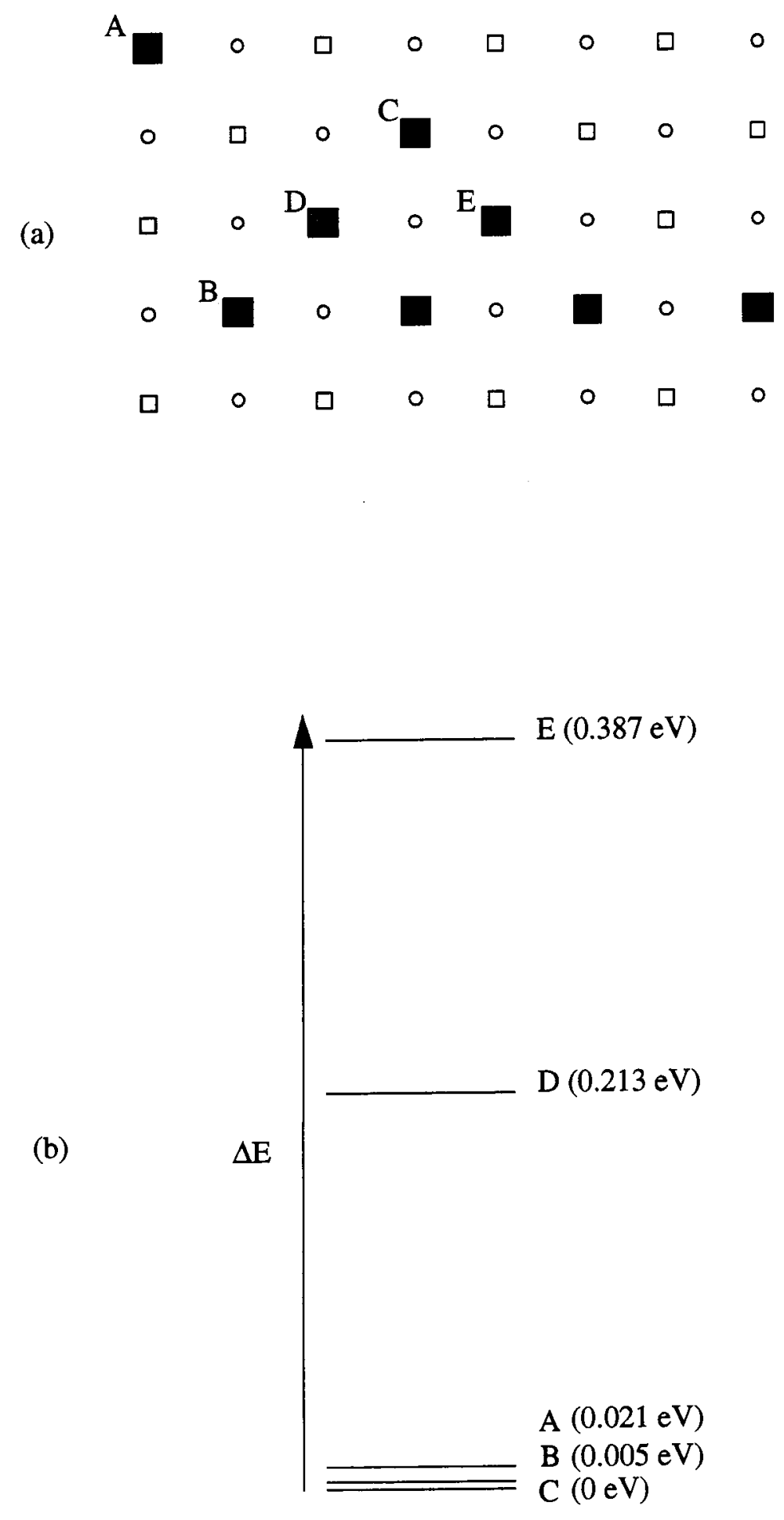

Fig. 5 
(a) $\Delta H=-0.0816 \mathrm{eV}$

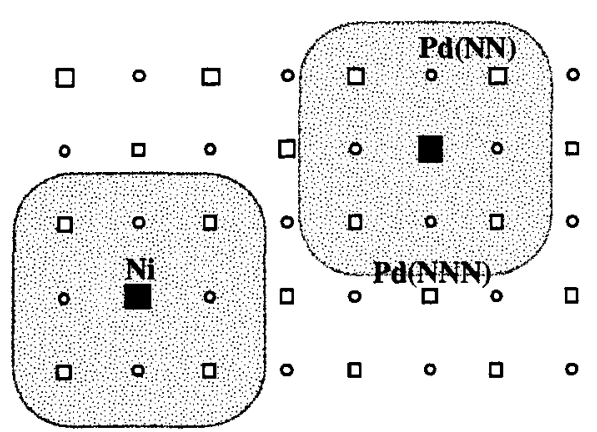

\begin{tabular}{lccccc}
\hline \multicolumn{5}{c}{ BFS energies per atom } \\
Atom & $m$ & $\varepsilon_{i}^{S}$ & $g_{i}$ & $\varepsilon_{i}^{C}-\varepsilon_{i}^{C_{0}}$ & $\varepsilon_{i}{ }^{T}$ \\
\hline $\mathrm{Ni}$ & 2 & 0.4406 & 0.5888 & -0.7749 & -0.0156 \\
$\operatorname{Pd}(\mathrm{NN})$ & 24 & 0 & 1 & -0.0020 & -0.0020 \\
$\operatorname{Pd}(\mathrm{NNN})$ & 12 & 0 & 1 & -0 & $\sim 0$ \\
\hline
\end{tabular}

(b) $\Delta H=-0.0967 \mathrm{eV}$

\begin{tabular}{lccccc}
\hline & \multicolumn{5}{c}{ BFS energies per atom } \\
Atom & $m$ & $\varepsilon_{i}^{S}$ & $g_{i}$ & $\varepsilon_{i}^{C}-\varepsilon_{i}^{C_{0}}$ & $\varepsilon_{i}^{T}$ \\
\hline $\mathrm{Ni}$ & 2 & 0.4406 & 0.5888 & -0.7739 & -0.0150 \\
$\operatorname{Pd}(\mathrm{NN})_{1}$ & 16 & 0 & 1 & -0.0020 & -0.0020 \\
$\operatorname{Pd}(\mathrm{NN})_{2}$ & 4 & 0 & 1 & -0.0085 & -0.0085 \\
$\operatorname{Pd}(\mathrm{NNN})$ & 10 & 0 & 1 & $\sim 0$ & $\sim 0$ \\
\hline
\end{tabular}

Fig. 6 